\title{
Severe Hypoglycemia and Risks of Vascular Events and Death
}

\author{
Sophia Zoungas, M.D., Ph.D., Anushka Patel, M.D., Ph.D., \\ John Chalmers, M.D., Ph.D., Bastiaan E. de Galan, M.D., Ph.D., \\ Qiang Li, M.Biostat., Laurent Billot, M.Sc., Mark Woodward, Ph.D., \\ Toshiharu Ninomiya, M.D., Ph.D., Bruce Neal, M.D., Ph.D., \\ Stephen MacMahon, D.Sc., Ph.D., Diederick E. Grobbee, M.D., Ph.D., \\ Andre Pascal Kengne, M.D., Ph.D., Michel Marre, M.D., Ph.D., \\ and Simon Heller, M.D., for the ADVANCE Collaborative Group
}

ABSTRACT

From the George Institute for International Health, University of Sydney, Sydney (S.Z., A.P., J.C., B.E.G., Q.L.M., L.B., M.W., T.N., B.N., S.M., A.P.K.); the School of Public Health, Monash University, Melbourne, Australia (S.Z.); the Department of General Internal Medicine, Radboud University Nijmegen Medical Center, Nijmegen (B.E.G.), and the Julius Center for Health Sciences and Primary Care, University Medical Center Utrecht, Utrecht (D.E.G.) - both in the Netherlands; Mount Sinai School of Medicine, New York (M.W.); Hôpital Bichat-Claude Bernard and Université Paris, Paris (M.M.); and the University of Sheffield and Shef field Teaching Hospitals National Health Service Foundation Trust, Sheffield, United Kingdom (S.H.). Address reprint requests to Dr. Zoungas at the George Institute for International Health, University of Sydney, P.O. Box M201, Missenden Rd., Sydney, NSW 2050, Australia, or at szoungas@ george.org.au.

N Engl J Med 2010;363:1410-8. Coppright (๑) 2010 Massachusetts Medical Society.

\section{BACKGROUND}

Severe hypoglycemia may increase the risk of a poor outcome in patients with type 2 diabetes assigned to an intensive glucose-lowering intervention. We analyzed data from a large study of intensive glucose lowering to explore the relationship between severe hypoglycemia and adverse clinical outcomes.

\section{METHODS}

We examined the associations between severe hypoglycemia and the risks of macrovascular or microvascular events and death among 11,140 patients with type 2 diabetes, using Cox proportional-hazards models with adjustment for covariates measured at baseline and after randomization.

\section{RESULTS}

During a median follow-up period of 5 years, 231 patients (2.1\%) had at least one severe hypoglycemic episode; 150 had been assigned to intensive glucose control $(2.7 \%$ of the 5571 patients in that group), and 81 had been assigned to standard glucose control (1.5\% of the 5569 patients in that group). The median times from the onset of severe hypoglycemia to the first major macrovascular event, the first major microvascular event, and death were 1.56 years (interquartile range, 0.84 to 2.41), 0.99 years (interquartile range, 0.40 to 2.17), and 1.05 years (interquartile range, 0.34 to 2.41), respectively. During follow-up, severe hypoglycemia was associated with a significant increase in the adjusted risks of major macrovascular events (hazard ratio, 2.88; 95\% confidence interval [CI], 2.01 to 4.12), major microvascular events (hazard ratio, $1.81 ; 95 \%$ CI, 1.19 to 2.74 ), death from a cardiovascular cause (hazard ratio, 2.68; $95 \%$ CI, 1.72 to 4.19 ), and death from any cause (hazard ratio, 2.69; 95\% CI, 1.97 to 3.67$)(\mathrm{P}<0.001$ for all comparisons). Similar associations were apparent for a range of nonvascular outcomes, including respiratory, digestive, and skin conditions ( $\mathrm{P}<0.01$ for all comparisons). No relationship was found between repeated episodes of severe hypoglycemia and vascular outcomes or death.

\section{CONCLUSIONS}

Severe hypoglycemia was strongly associated with increased risks of a range of adverse clinical outcomes. It is possible that severe hypoglycemia contributes to adverse outcomes, but these analyses indicate that hypoglycemia is just as likely to be a marker of vulnerability to such events. (Funded by Servier and the National Health and Medical Research Council of Australia; ClinicalTrials.gov number, NCT00145925.) 
I

$\mathrm{N}$ PATIENTS WITH DIABETES TREATED WITH insulin or insulin secretagogues, severe hypoglycemia is more common when glucose control is intensified. ${ }^{1-4}$ Although most episodes of severe hypoglycemia resolve without apparent permanent injury, there are anecdotal reports of acute coronary syndromes coinciding with hypoglycemia in people with type 2 diabetes. ${ }^{5,6}$ In addition, observational studies have suggested that hypoglycemia and reduced levels of glycated hemoglobin are associated with an increased risk of death in patients with diabetes or hyperglycemia who have been hospitalized for myocardial infarction. ${ }^{7-11}$

Recently completed trials investigating the effect of intensive glucose control on macrovascular outcomes in patients with long-standing type 2 diabetes have individually failed to demonstrate clear reductions in cardiovascular events or mortality. ${ }^{1-3}$ A meta-analysis showed that intensive glucose control reduced the risk of myocardial infarction by $15 \%$, with no adverse effect on the risk of death, but that it simultaneously increased the risk of severe hypoglycemia. ${ }^{12}$ Nonetheless, the excess mortality observed with intensive glucose control in the Action to Control Cardiovascular Risk in Diabetes (ACCORD) study has fueled speculation about the adverse effects of such regimens in patients with type 2 diabetes. ${ }^{13}$ Post hoc analyses of ACCORD study data suggest that the excess mortality in the intensively treated group was not directly explained by the high rate of hypoglycemia. ${ }^{14}$

We examined the relationship between severe hypoglycemia and the subsequent risks of vascular complications and death among 11,140 patients with type 2 diabetes who participated in the Action in Diabetes and Vascular Disease: Preterax and Diamicron Modified Release Controlled Evaluation (ADVANCE) study (ClinicalTrials.gov number, NCT00145925).

\section{METHODS}

\section{STUDY DESIGN AND OVERSIGHT}

ADVANCE was a factorial trial with two randomized comparisons: a double-blind assessment of the efficacy of perindopril-indapamide in lowering blood pressure, as compared with placebo, and an open-label assessment of the effects of intensive glucose lowering with the use of a modifiedrelease formulation of gliclazide as compared with standard, guideline-based glucose lowering on the risks of vascular outcomes and death among patients with type 2 diabetes. Approval for the trial was obtained from the ethics committee of each study center, and all participants provided written informed consent. ${ }^{3,15}$ The main results for each comparison have been reported previously. 3,15

\section{STUDY POPULATION}

A total of 11,140 patients who were at least 55 years of age were recruited for the study from 215 centers in 20 countries between June 2001 and March 2003. Eligible patients had received a diagnosis of type 2 diabetes after the age of 30 years and had a history of major macrovascular or microvascular disease or at least one other cardiovascular risk factor. ${ }^{3}$ No threshold for level of blood glucose or glycated hemoglobin was specified for inclusion in the study, although patients with a clear indication for long-term insulin use at baseline were ineligible. Patients assigned to the intensive glucose-lowering intervention were given modified-release gliclazide (Diamicron MR, Servier) and other glucose-lowering drugs as required, with a target glycated hemoglobin level of $6.5 \%$ or less. ${ }^{3}$ Weight, height, blood pressure, and levels of glycated hemoglobin and serum creatinine were measured at baseline, at 4 months, and every 6 months thereafter. ${ }^{3}$

\section{DEFINITION OF HYPOGLYCEMIA}

Hypoglycemia was defined as a blood glucose level of less than $2.8 \mathrm{mmol}$ per liter (50 mg per deciliter) or the presence of typical symptoms and signs of hypoglycemia without other apparent cause. Patients with transient dysfunction of the central nervous system who were unable to treat themselves (requiring help from another person) were considered to have severe hypoglycemia. Episodes of severe hypoglycemia were reported at the time of their occurrence during study follow-up, with a full description of the event. Each report was reviewed to confirm that the criteria were met. Patients with transient dysfunction of the central nervous system who were able to treat themselves were considered to have minor hypoglycemia. Episodes of minor hypoglycemia were reported at follow-up visits.

\section{CLINICAL OUTCOMES}

The primary clinical outcomes considered were the first major macrovascular event (death from a cardiovascular cause, nonfatal myocardial infarction, or nonfatal stroke) and the first major micro- 
vascular event (new or worsening nephropathy or retinopathy). The secondary outcomes considered were death from any cause and death from a cardiovascular event. Primary and secondary outcomes were validated by an independent adjudication committee whose members were unaware of the treatment assignments. ${ }^{3}$ Nonvascular clinical outcomes were reported during follow-up as serious adverse events and coded according to the International Classification of Diseases, 9th Revision.

\section{STATISTICAL ANALYSIS}

A full description of the statistical analysis is provided in the Supplementary Appendix, available with the full text of this article at NEJM.org. In brief, rates of severe and minor hypoglycemia during follow-up were estimated for the entire cohort and according to treatment assignments. Baseline risk factors for severe hypoglycemia were examined with the use of univariate and multivariateadjusted Cox proportional regression models. Incidence rates of clinical outcomes according to hypoglycemic status were examined in the entire cohort. Crude, annualized mortality rates within each glucose treatment group (intensive glucose control and standard glucose control) for patients who did and those who did not report severe hypoglycemia were determined by dividing the number of deaths by the cumulative time at risk.

Associations between severe hypoglycemia and clinical outcomes were tested with the use of timedependent Cox proportional-hazards models of the time from randomization to the first clinical outcome. Models were adjusted for all baseline and time-dependent covariates (variables measured during follow-up) that were thought to be potential confounders, as listed in the Supplementary Appendix. For each clinical outcome, the followup time for every patient was divided into as many intervals as there were distinct event times in the whole cohort. Each interval included the latest measured value for each time-dependent covariate and a variable indicating whether the patient had the clinical outcome of interest during that interval. ${ }^{16}$ Follow-up for each participant was considered separately for each major outcome and ended on the date of the first clinical event, the date of death, or the date of data censoring at study completion.

The primary analysis assumed that patients were exposed to hypoglycemia from the time of the first severe hypoglycemic episode until the end of follow-up or the occurrence of a clinical event.
Additional sensitivity analyses tested various exposure times, use of logistic-regression models, and additional adjustment for cardiovascular events occurring after randomization, as described in the Supplementary Appendix. Model predictive capacity, with and without severe hypoglycemia, was tested with the use of C statistics, ${ }^{17}$ the integrateddiscrimination-improvement statistic, ${ }^{18}$ and the Hosmer-Lemeshow chi-square statistic. ${ }^{19}$ Analyses were performed with the use of SAS statistical software, version 9.1 (SAS Institute). All statistical tests were two-sided, and a P value of less than 0.05 was considered to indicate statistical significance, but all results were interpreted in light of the many comparisons made.

\section{RESULTS}

\section{RATES OF HYPOGLYCEMIA OVER TIME}

During a median follow-up period of 5 years, 231 of 11,140 study patients (2.1\%) reported 299 severe hypoglycemic events: 150 patients in the intensive-intervention group (2.7\%) reported 195 events, and 81 patients in the standard-intervention group (1.5\%) reported 104 events (Table 1). Over time, the rate of severe hypoglycemia increased in the group undergoing intensive glucose control ( $\mathrm{P}<0.001$ for trend) but remained relatively stable in the group receiving standard treatment ( $\mathrm{P}=0.38$ for trend) (Fig. 1A). A total of $4975 \mathrm{pa}-$ tients $(44.7 \%)$ reported minor hypoglycemia during follow-up; 2898 of these patients (52.0\%) had been assigned to intensive control and 2077 (37.3\%) to standard control (Table 1).

\section{RISK FACTORS FOR SEVERE HYPOGLYCEMIA}

Univariate and multivariate analyses showed that the following variables were independent risk factors for severe hypoglycemia: older age, longer duration of diabetes, higher creatinine levels, lower body-mass index, lower cognitive function, use of two or more oral glucose-lowering drugs, history of smoking or microvascular disease, and assignment to intensive glucose control $(\mathrm{P}<0.05$ for all comparisons; for details, see Table 1 in the Supplementary Appendix). When these analyses were stratified according to treatment group, the risk factors for severe hypoglycemia were similar.

\section{ASSOCIATION OF SEVERE HYPOGLYCEMIA} WITH VASCULAR OUTCOMES AND DEATH

During follow-up, 2125 patients had a major macrovascular or microvascular event, 87 of whom re- 


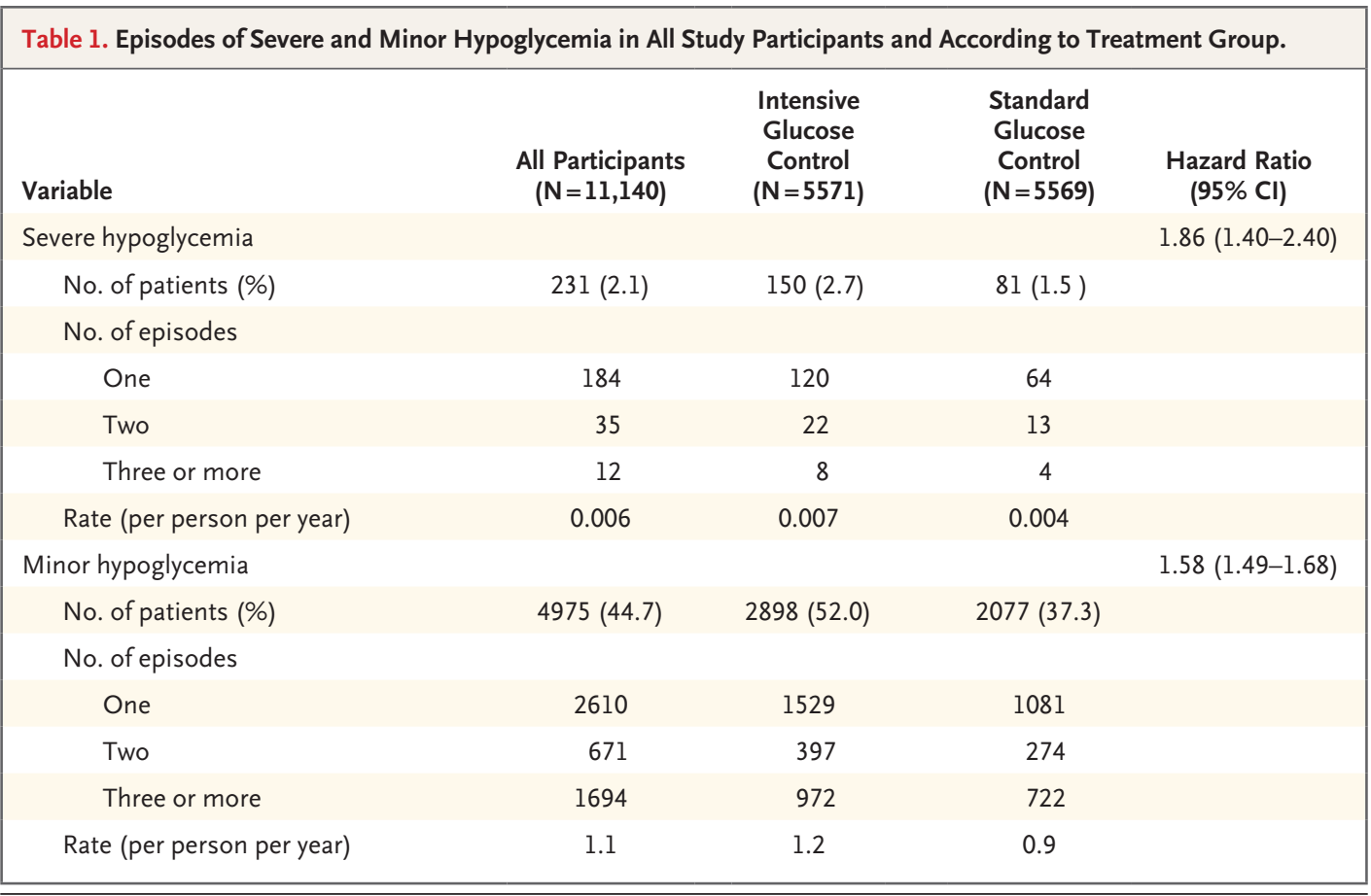

ported severe hypoglycemia (before the event in 40 patients and after the event in 47), and 1031 patients died, 45 of whom had reported severe hypoglycemia. The median times from the episode of severe hypoglycemia to the subsequent first major macrovascular or microvascular event were 1.56 years (interquartile range, 0.84 to 2.41 ) and 0.99 years (interquartile range, 0.40 to 2.17 ), respectively. The median time from severe hypoglycemia to death was 1.05 years (interquartile range, 0.34 to 2.41 ) - 1.31 years (interquartile range, 0.80 to 2.41) for death from a cardiovascular event and 0.74 years (interquartile range, 0.13 to 2.60 ) for death from a noncardiovascular cause (Fig. 1B).

Among patients who reported severe hypoglycemia, $16.8 \%$ (35 of 208) had a subsequent major macrovascular event, $11.5 \%$ (24 of 209) had a subsequent major microvascular event, and 19.5\% (45 of 231) died; the respective rates for those who did not report severe hypoglycemia were $10.2 \%$ (1112 of 10,932 patients), 10.1\% (1107 of 10,931), and $9.0 \%$ (986 of 10,909$)$, respectively. The proportions of patients who died from cardiovascular causes and from noncardiovascular causes were similar among patients reporting severe hypoglycemia (49\% and 51\%, respectively) and those not reporting severe hypoglycemia (53\% and $47 \%$, respectively; $\mathrm{P}=0.09$ ). For those reporting severe hypoglycemia, annual death rates were lower in the group receiving intensive treatment than in the group receiving standard treatment (3.6\% vs. 5.1\%). In contrast, for those not reporting severe hypoglycemia, annual death rates were similar in the two treatment groups $(1.8 \%$ with the intensive intervention and $1.9 \%$ with standard treatment) (Table 2). There was no evidence of interaction among severe hypoglycemia, the assigned glucoselowering treatment, and the risk of death $(\mathrm{P}=0.30)$.

The unadjusted risks of a major macrovascular event, a major microvascular event, death from any cause, and death from a cardiovascular or noncardiovascular cause were significantly increased among patients who had severe hypoglycemia as compared with those who did not (Table 3). After adjustment for a number of potential confounding variables measured at baseline or during follow-up, the associations were markedly attenuated but remained significant $(\mathrm{P}<0.01$ for all comparisons).

In analyses in which the period of follow-up after a severe hypoglycemic episode was limited to 3 months and to 6 months, the unadjusted and adjusted associations with all major macrovascular outcomes and death (from any cause or from a cardiovascular or noncardiovascular cause), remained significant (Table 3). The adjusted associations for major microvascular events were no longer significant when the period was limited to 3 months, but the point estimates of association were similar (Table 3). When the analyses were 


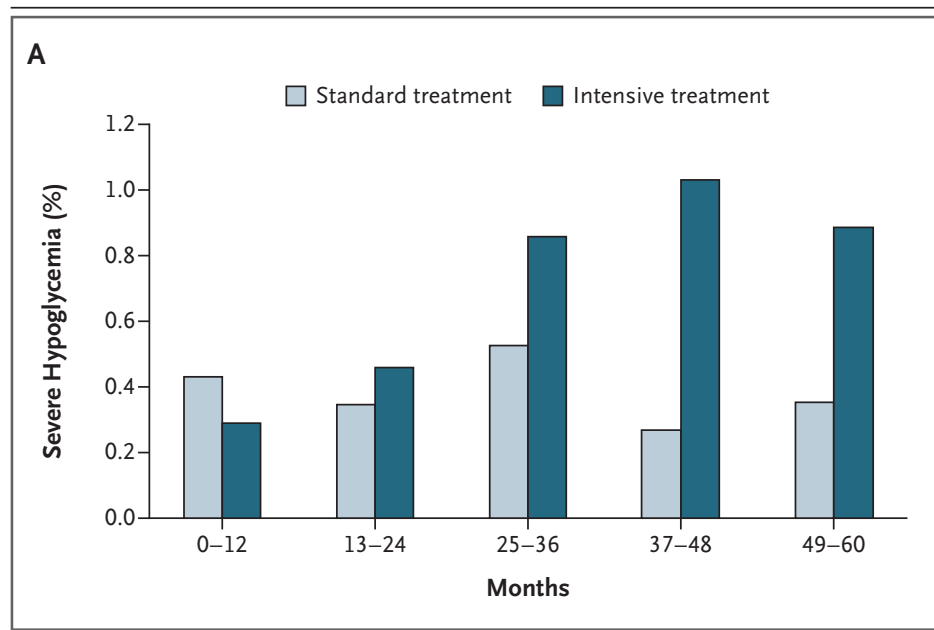

B

Macrovascular event $\square$ Microvascular event $\square$ Death from any cause

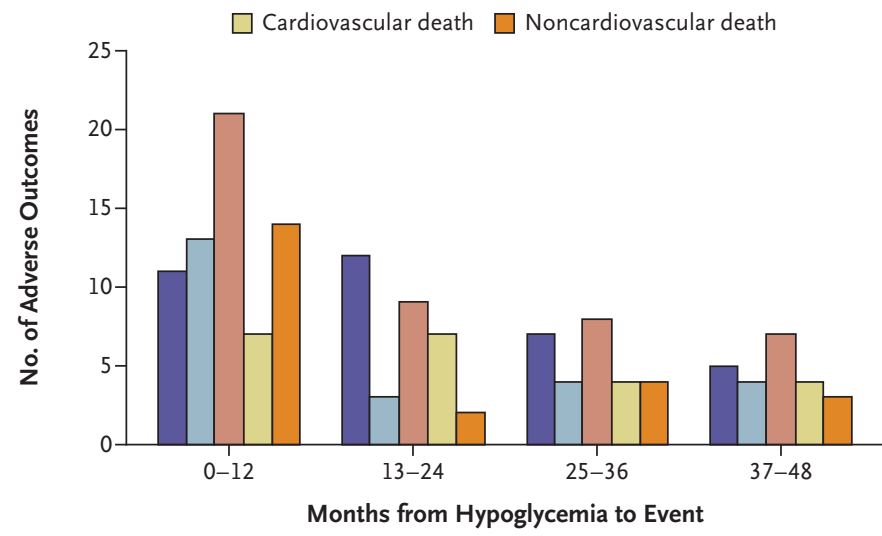

Figure 1. Annual Rates of Severe Hypoglycemia According to Treatment Assignment and Adverse Clinical Outcomes among Patients with Severe Hypoglycemia.

Panel A shows the rates of severe hypoglycemia in the group assigned to the intensive glucose-lowering intervention $(P<0.001$ for trend) and the group assigned to standard glucose control ( $P=0.38$ for trend). Panel $B$ shows the frequency of adverse clinical outcomes after the occurrence of a severe hypoglycemic episode. from any cause was 1.57 (95\% confidence interval [CI], 1.02 to 2.41) with a single severe hypoglycemic episode and 1.24 ( $95 \%$ CI, 0.58 to 2.68) with two or more episodes $(\mathrm{P}>0.10$ for the comparison of risk among those reporting two or more hypoglycemic episodes vs. those reporting one episode). When we examined the effect of adding the occurrence of severe hypoglycemia to models predicting absolute risks of vascular outcomes and death, we found no improvement in prediction for any outcome ( $\mathrm{P} \geq 0.05$ for all $\mathrm{C}$ statistics; for details, see Table 3 in the Supplementary Appendix).

\section{ASSOCIATION OF MINOR HYPOGLYCEMIA WITH VASCULAR OUTCOMES AND DEATH}

The risks of major macrovascular outcomes and death were reduced among patients reporting minor hypoglycemia as compared with those who did not report minor hypoglycemia (for details, see Table 4 in the Supplementary Appendix). However, there was an increased risk of major microvascular events (unadjusted odds ratio, 1.25; 95\% CI, 1.11 to 1.42 ; $\mathrm{P}<0.001$; adjusted odds ratio, 1.29; $95 \%$ CI, 1.13 to 1.48 ; $\mathrm{P}<0.001$ ).

\section{ASSOCIATION OF SEVERE HYPOGLYCEMIA WITH NONVASCULAR OUTCOMES}

During follow-up, disorders of the respiratory system, the digestive system, and the skin developed in some study participants, as did cancer (Fig. 2). The unadjusted risks of these nonvascular conditions were increased among patients who reported severe hypoglycemia as compared with those who did not (Fig. 2). After adjustment, the associations remained significant for all outcomes except cancer ( $\mathrm{P}<0.05$ for all comparisons) (Fig. 2).

\section{ADDITIONAL SENSITIVITY ANALYSES}

The observed associations between severe hypoglycemia and the risks of major vascular events and death were robust on examination with pooled logistic regression. For the risk of death from any cause, further adjustment for cardiovascular events occurring after randomization resulted in additional attenuation of the odds ratio, from 2.61 (95\% CI, 1.74 to 3.93 ) to 1.34 (95\% CI, 0.79 to 2.27).

\section{DISCUSSION} lationship between repeated episodes of severe hypoglycemia and vascular outcomes or death, although few patients had recurrent events (for details, see Table 2 in the Supplementary Appendix). For example, the adjusted odds ratio for death
In the ADVANCE study, severe hypoglycemia was clearly associated with increased risks of macrovascular events, microvascular events, and death 


\begin{tabular}{|c|c|c|c|}
\hline \multirow[t]{2}{*}{ Severe Hypoglycemia } & \multicolumn{2}{|c|}{ Deaths $(N=1031)$} & \multirow[t]{2}{*}{$\begin{array}{c}\text { Unadjusted } \\
\text { Hazard Ratio }(95 \% \mathrm{Cl})\end{array}$} \\
\hline & Intensive Glucose Control & Standard Glucose Control & \\
\hline \multicolumn{4}{|c|}{ no. of deaths/no. of person- $\gamma r$ (\% per $y r)$} \\
\hline No episodes & $472 / 26,034(1.8)$ & $514 / 26,392(1.9)$ & $0.93(0.82-1.06)$ \\
\hline One or more episodes & $26 / 718(3.6)$ & $19 / 369(5.1)$ & $0.67(0.37-1.21)$ \\
\hline
\end{tabular}

from both cardiovascular and noncardiovascular causes. Risks were also increased for a range of nonvascular events that included cancer and conditions affecting the respiratory system, the digestive system, and the skin. The association of hypoglycemia with increased risks of vascular events, nonvascular events, and death were similar for patients assigned to standard glucose control and those assigned to intensive glucose control, and although it was attenuated after adjustment for potential confounding factors, the association remained significant for most outcomes. These findings in a community-dwelling population of patients reporting severe hypoglycemia are supported by other data, ${ }^{8-11,20,21}$ including a recent report from the ACCORD study group. ${ }^{14}$

Plausible mechanisms by which hypoglycemia might cause cardiovascular disease or lead to death from cardiovascular disease include sympathoadrenal activation, abnormal cardiac repolarization, increased thrombogenesis, inflammation, and vasoconstriction. ${ }^{22}$ In a recent study involving healthy volunteers, two induced episodes of mild hypoglycemia led to impaired cardiac autonomic function the day after each episode. ${ }^{23}$ Although it is unclear whether such effects persist, impaired cardiac autonomic function is associated with increased mortality among patients at high cardiovascular risk, ${ }^{24-26}$ including those with diabetes. ${ }^{27}$ We did not measure autonomic function and therefore could not examine its contributory effects. It is possible that episodes of asymptomatic or unreported symptomatic hypoglycemia followed the initial occurrence of severe hypoglycemia and thus contributed to the increased risk of subsequent events. Patients who have repeated hypoglycemic episodes often have decreased awareness of hypoglycemia, ${ }^{28}$ and lack of awareness might explain the absence of any clear temporal link between severe hypoglycemia and the adverse outcomes observed in our analyses.
It is also possible that severe hypoglycemia reflects the effects of coexisting conditions and unmeasured or incompletely quantified confounding variables and is a marker of an increased risk of adverse clinical outcomes rather than a direct cause. ${ }^{29}$ The marked attenuation of the risk estimates after adjustment for a number of baseline and time-dependent characteristics of the patients suggests that the effects of confounding were substantial. The presence of coexisting conditions could increase a patient's vulnerability to both severe hypoglycemia and an adverse clinical outcome in the absence of a direct causal link between the two. For example, the observed association between severe hypoglycemia and microvascular disease may be more easily explained by the presence of risk factors common to both (e.g., older age and longer duration of diabetes) than by a direct effect of hypoglycemia on the occurrence of nephropathy or retinopathy. In addition, the shorter interval between a reported episode of severe hypoglycemia and death than between such an episode and a macrovascular event suggests that among patients with severe hypoglycemia, those who died were sicker at the time of their episode of hypoglycemia than those who had other types of events.

In both the ADVANCE study and the ACCORD study, ${ }^{14}$ annual mortality among patients who reported severe hypoglycemia was higher in the group receiving standard treatment than in the group receiving intensive treatment. Despite the higher rate of severe hypoglycemia in the latter group, hypoglycemia was not considered to be a likely explanation for the excess mortality associated with intensive control in the ACCORD trial. In the ADVANCE study, intensive glucose control was not associated with excess mortality. In conjunction with the absence of a clear dose-response relationship between repeated episodes of hypoglycemia and subsequent macrovascular events or 


\begin{tabular}{|c|c|c|c|c|c|}
\hline $\begin{array}{c}\text { Clinical Outcome and Interval } \\
\text { after Hypoglycemia }\end{array}$ & No. of Events & $\begin{array}{c}\text { Hazard Ratio } \\
\text { Adjusted for } \\
\text { Treatment Assignment } \\
(95 \% \mathrm{Cl})\end{array}$ & P Value & $\begin{array}{c}\text { Hazard Ratio } \\
\text { Adjusted for } \\
\text { Multiple Covariates } \\
(95 \% \mathrm{Cl}){ }^{\dagger}\end{array}$ & P Value \\
\hline Macrovascular events & 1147 & $4.05(2.86-5.74)$ & $<0.001$ & $3.45(2.34-5.08)$ & $<0.001$ \\
\hline $3 \mathrm{mo}$ & & $3.27(1.22-8.73)$ & 0.02 & $3.55(1.32-9.54)$ & 0.01 \\
\hline $6 \mathrm{mo}$ & & $2.61(1.17-5.83)$ & 0.02 & $2.75(1.22-6.19)$ & 0.01 \\
\hline Microvascular events & 1131 & $2.39(1.60-3.59)$ & $<0.001$ & $2.07(1.32-3.26)$ & $<0.001$ \\
\hline $3 \mathrm{mo}$ & & $2.90(1.09-7.74)$ & 0.03 & $1.96(0.63-6.17)$ & 0.24 \\
\hline $6 \mathrm{mo}$ & & $3.24(1.62-6.50)$ & $<0.001$ & $2.41(1.07-5.43)$ & 0.03 \\
\hline Death from any cause & 1031 & $4.86(3.60-6.57)$ & $<0.001$ & $3.30(2.31-4.72)$ & $<0.001$ \\
\hline $3 \mathrm{mo}$ & & $10.4(6.02-18.00)$ & $<0.001$ & $5.86(2.97-11.60)$ & $<0.001$ \\
\hline $6 \mathrm{mo}$ & & $7.28(4.50-11.80)$ & $<0.001$ & $4.28(2.36-7.75)$ & $<0.001$ \\
\hline Death from cardiovascular cause & 542 & $4.87(3.17-7.49)$ & $<0.001$ & $3.78(2.34-6.11)$ & $<0.001$ \\
\hline $3 \mathrm{mo}$ & & $6.25(2.34-6.70)$ & $<0.001$ & $5.35(1.94-14.80)$ & $<0.01$ \\
\hline $6 \mathrm{mo}$ & & $4.20(1.74-10.10)$ & $<0.01$ & $3.57(1.43-8.90)$ & 0.01 \\
\hline Death from noncardiovascular cause & 489 & $4.82(3.16-7.35)$ & $<0.001$ & $2.86(1.67-4.90)$ & $<0.001$ \\
\hline $3 \mathrm{mo}$ & & $14.20(7.35-27.60)$ & $<0.001$ & $6.19(2.47-15.50)$ & $<0.001$ \\
\hline $6 \mathrm{mo}$ & & $10.30(5.78-18.20)$ & $<0.001$ & $4.95(2.26-10.80)$ & $<0.001$ \\
\hline \multicolumn{6}{|c|}{$\begin{array}{l}\text { Severe hypoglycemia was defined as a blood glucose level of less than } 2.8 \mathrm{mmol} \text { per liter ( }<50 \mathrm{mg} \text { per deciliter), with transient dysfunction } \\
\text { of the central nervous system, without other apparent cause, during which the patient was unable to administer treatment (requiring help } \\
\text { from another person). } \\
\text { Covariates included sex, duration of diabetes, treatment assignment, presence or absence of a history of macrovascular disease, presence } \\
\text { or absence of a history of microvascular disease, and smoking status at baseline. Time-dependent covariates during follow-up included age; } \\
\text { level of glycated hemoglobin; body-mass index; creatinine level; ratio of urinary albumin to creatinine; systolic blood pressure; use or non- } \\
\text { use of sulfonylurea, metformin, thiazolidinedione, insulin, or any other diabetes drug; and use or nonuse of antihypertensive agents. }\end{array}$} \\
\hline
\end{tabular}

death, these observations add to the uncertainty about the direct causal relationship between hypoglycemia and death and between hypoglycemia and vascular outcomes.

Our study is among the largest to examine the association of severe hypoglycemia with macrovascular and microvascular events and death from cardiovascular and noncardiovascular causes. The substantial size of the cohort and the relatively large numbers of events recorded allowed us to examine associations with temporal and statistical precision. Severe hypoglycemic events and clinical outcomes were systematically reported as serious adverse events and were subject to rigorous independent review. However, the number of patients reporting severe hypoglycemia was relatively small. In addition, the occurrence of severe hypoglycemia before study entry and the presence of autonomic neuropathy were not recorded. Episodes of hypoglycemia that may have contributed to adverse outcomes but that did not meet the definition of severe hypoglycemia may have been unreported. In addition, patients and their care providers may have been unaware of minor hypoglycemic episodes. Given these considerations, the reliability of the measurement of the total hypoglycemic burden remains somewhat uncertain.

In conclusion, our study shows that severe hypoglycemia is strongly associated with increased risks of a broad range of adverse clinical outcomes in patients with long-standing type 2 diabetes, including vascular events and death. Neither a close temporal relationship nor a dose-response relationship was observed. Although our findings cannot exclude the possibility that severe hypoglycemia has a direct causal link with these outcomes, they suggest that it is as likely to be a marker of vulnerability to a wide range of adverse clinical outcomes. In either case, the presence of 


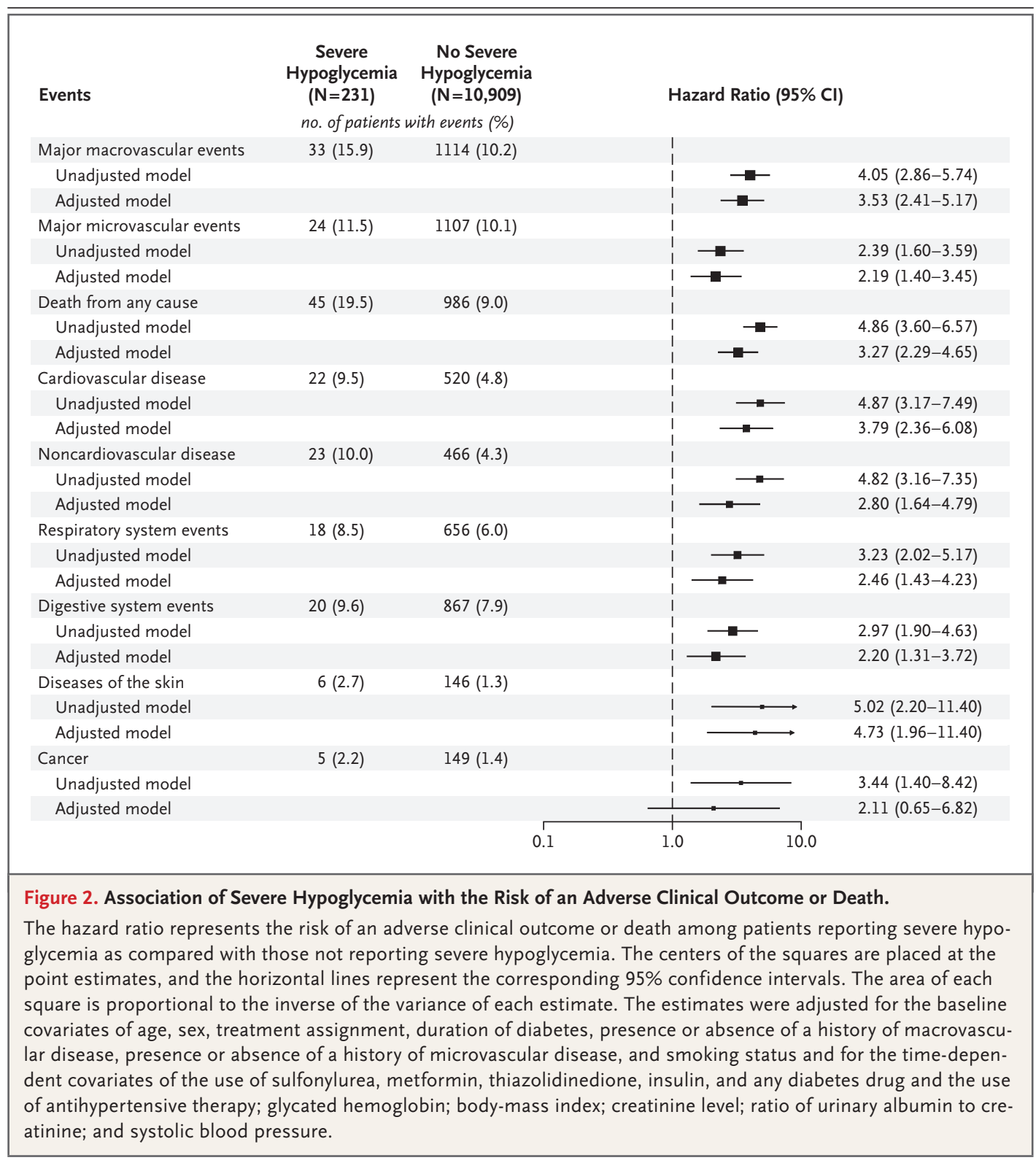

severe hypoglycemia should raise clinical suspicion of the patient's susceptibility to adverse outcomes and prompt action to address this possibility.
Supported by grants from Servier and the National Health and Medical Research Council of Australia.

Disclosure forms provided by the authors are available with the full text of this article at NEJM.org.

\section{REFERENCES}

1. Duckworth W, Abraira C, Moritz T, et al. Glucose control and vascular complications in veterans with type 2 diabetes. N Engl J Med 2009;360:129-39. [Erratum, N Engl J Med 2009;361:1028.]

2. The Action to Control Cardiovascular Risk in Diabetes Study Group. Effects of intensive glucose lowering in type 2 diabetes. N Engl J Med 2008;358:2545-59.

3. The ADVANCE Collaborative Group. Intensive blood glucose control and vascular outcomes in patients with type 2 diabetes. N Engl J Med 2008;358:2560-72.

4. United Kingdom Prospective Diabetes
Study Group. United Kingdom Prospective Diabetes Study 24: a 6-year, randomized, controlled trial comparing sulfonylurea, insulin, and metformin therapy in patients with newly diagnosed type 2 diabetes that could not be controlled with diet therapy. Ann Intern Med 1998;128:165-75. 
5. Desouza C, Salazar H, Cheong B, Murgo J, Fonseca V. Association of hypoglycemia and cardiac ischemia: a study based on continuous monitoring. Diabetes Care 2003;26:1485-9.

6. Duh E, Feinglos M. Hypoglycemiainduced angina pectoris in a patient with diabetes mellitus. Ann Intern Med 1994; 121:945-6.

7. Currie CJ, Peters JR, Tynan A, et al. Survival as a function of $\mathrm{HbA}(1 \mathrm{c})$ in people with type 2 diabetes: a retrospective cohort study. Lancet 2010;375:481-9.

8. Kosiborod M, Inzucchi SE, Goyal A, et al. Relationship between spontaneous and iatrogenic hypoglycemia and mortality in patients hospitalized with acute myocardial infarction. JAMA 2009;301:1556-64.

9. Kosiborod M, Inzucchi SE, Krumholz HM, et al. Glucometrics in patients hospitalized with acute myocardial infarction: defining the optimal outcomes-based measure of risk. Circulation 2008;117:1018-27. 10. Pinto DS, Skolnick AH, Kirtane AJ, et al. U-shaped relationship of blood glucose with adverse outcomes among patients with ST-segment elevation myocardial infarction. J Am Coll Cardiol 2005;46:178-80. 11. Svensson AM, McGuire DK, Abrahamsson P, Dellborg M. Association between hyper- and hypoglycaemia and 2 year allcause mortality risk in diabetic patients with acute coronary events. Eur Heart J 2005;26:1255-61.

12. Turnbull FM, Abraira C, Anderson RJ, et al. Intensive glucose control and macrovascular outcomes in type 2 diabetes. Diabetologia 2009;52:2288-98. [Erratum, Diabetologia 2009;52:2470.]

13. Skyler JS, Bergenstal R, Bonow RO, et al. Intensive glycemic control and the prevention of cardiovascular events: implications of the ACCORD, ADVANCE, and VA
Diabetes Trials: a position statement of the American Diabetes Association and a scientific statement of the American College of Cardiology Foundation and the American Heart Association. J Am Coll Cardiol 2009;53:298-304.

14. Bonds DE, Miller ME, Bergenstal RM, et al. The association between symptomatic, severe hypoglycaemia and mortality in type 2 diabetes: retrospective epidemiological analysis of the ACCORD study. BMJ 2010;340:b4909.

15. Patel A, MacMahon S, Chalmers J, et al. Effects of a fixed combination of perindopril and indapamide on macrovascular and microvascular outcomes in patients with type 2 diabetes mellitus (the ADVANCE trial): a randomised controlled trial. Lancet 2007;370:829-40.

16. Therneau TM, Grambsch PM. Modelling survival data: extending the Cox model. New York: Springer, 2000.

17. DeLong ER, DeLong DM, Clarke-Pearson DL. Comparing the areas under two or more correlated receiver operating characteristic curves: a nonparametric approach. Biometrics 1988:44:837-45.

18. Pencina MJ, D'Agostino RB, D'Agostino RB Jr, Vasan RS. Evaluating the added predictive ability of a new marker: from area under the ROC curve to reclassification and beyond. Stat Med 2008;27:157-72, 207-12.

19. Hosmer DW, Hosmer T, Le Cessie S, Lemeshow S. A comparison of goodnessof-fit tests for the logistic regression model. Stat Med 1997;16:965-80.

20. Hoekstra JBL, Hermanides J, Bosman $\mathrm{RJ}$, et al. Hypoglycaemia is related to mortality in the ICU. Diabetologia 2009;52: Suppl 1:237. abstract.

21. Miller ME, Fincke G, Lafrance JP, et al. Hypoglycaemia and risk of myocardial infarction in U.S. veterans with diabetes. Diabetologia 2009;52:Suppl 1:63. abstract. 22. Wright RJ, Frier BM. Vascular disease and diabetes: is hypoglycaemia an aggravating factor? Diabetes Metab Res Rev 2008;24:353-63.

23. Adler GK, Bonyhay I, Failing H, Waring $\mathrm{E}$, Dotson S, Freeman R. Antecedent hypoglycemia impairs autonomic cardiovascular function: implications for rigorous glycemic control. Diabetes 2009;58: 360-6.

24. Bigger JT, Fleiss JL, Rolnitzky LM, Steinman RC. The ability of several shortterm measures of $R R$ variability to predict mortality after myocardial infarction. Circulation 1993;88:927-34.

25. De Ferrari GM, Sanzo A, Bertoletti A, Specchia G, Vanoli E, Schwartz PJ. Baroreflex sensitivity predicts long-term cardiovascular mortality after myocardial infarction even in patients with preserved left ventricular function. J Am Coll Cardiol 2007;50:2285-90.

26. La Rovere MT, Bigger JT Jr, Marcus FI, Mortara A, Schwartz PJ. Baroreflex sensitivity and heart-rate variability in prediction of total cardiac mortality after myocardial infarction. Lancet 1998;351:478-84. 27. Maser RE, Mitchell BD, Vinik AI, Freeman $\mathrm{R}$. The association between cardiovascular autonomic neuropathy and mortality in individuals with diabetes: a meta-analysis. Diabetes Care 2003;26:1895-901.

28. Cryer PE. Mechanisms of hypoglycemia-associated autonomic failure and its component syndromes in diabetes. Diabetes 2005;54:3592-601.

29. MacMahon S, Collins R. Reliable assessment of the effects of treatment on mortality and major morbidity. II. Observational studies. Lancet 2001;357:455-62. Coppright (๑) 2010 Massachusetts Medical Society. 